\title{
In Silico Design of Human IMPDH Inhibitors Using Pharmacophore Mapping and Molecular Docking Approaches
}

\author{
Rui-Juan Li, ${ }^{1}$ Ya-Li Wang, ${ }^{2}$ Qing-He Wang, ${ }^{1}$ Jian Wang, ${ }^{1}$ and Mao-Sheng Cheng ${ }^{1}$ \\ ${ }^{1}$ Key Laboratory of Structure-Based Drug Design \& Discovery of Ministry of Education, School of Pharmaceutical Engineering, \\ Shenyang Pharmaceutical University, Shenyang 110016, China \\ ${ }^{2}$ Institute of Medicinal Biotechnology, Chinese Academy of Medical Science and Peking Union Medical College, Beijing 10050, China
}

Correspondence should be addressed to Jian Wang; jianwang@syphu.edu.cn and Mao-Sheng Cheng; mscheng@syphu.edu.cn

Received 20 November 2014; Revised 8 January 2015; Accepted 20 January 2015

Academic Editor: John Mitchell

Copyright (C) 2015 Rui-Juan Li et al. This is an open access article distributed under the Creative Commons Attribution License, which permits unrestricted use, distribution, and reproduction in any medium, provided the original work is properly cited.

\begin{abstract}
Inosine $5^{\prime}$-monophosphate dehydrogenase (IMPDH) is one of the crucial enzymes in the de novo biosynthesis of guanosine nucleotides. It has served as an attractive target in immunosuppressive, anticancer, antiviral, and antiparasitic therapeutic strategies. In this study, pharmacophore mapping and molecular docking approaches were employed to discover novel Homo sapiens IMPDH (hIMPDH) inhibitors. The Güner-Henry (GH) scoring method was used to evaluate the quality of generated pharmacophore hypotheses. One of the generated pharmacophore hypotheses was found to possess a GH score of 0.67 . Ten potential compounds were selected from the ZINC database using a pharmacophore mapping approach and docked into the IMPDH active site. We find two hits (i.e., ZINC02090792 and ZINC00048033) that match well the optimal pharmacophore features used in this investigation, and it is found that they form interactions with key residues of IMPDH. We propose that these two hits are lead compounds for the development of novel hIMPDH inhibitors.
\end{abstract}

\section{Introduction}

Inosine $5^{\prime}$-monophosphate dehydrogenase (IMPDH) is a rate-limiting enzyme in the de novo synthesis of guanine nucleotides. It catalyzes the conversion of inosine $5^{\prime}$-monophosphate (IMP) to xanthosine $5^{\prime}$-monophosphate (XMP) $[1,2]$ and therefore plays an important role in the regulation of cell growth [3]. There are two isoforms of Homo sapiens IMPDH (hIMPDH), labeled types I and II, which share $84 \%$ amino acid identity. hIMPDH type I (hIMPDH1) is the main species in normal leukocytes and hIMPDH type II (hIMPDH2) predominates over hIMPDH1 in the tumor cells and activated peripheral blood lymphocytes [4-8]. Gene sequence variation in the hIMPDH2 gene may contribute to the large interindividual difference of baseline hIMPDH enzyme activity, immunosuppressive efficacy, and side effects in transplant recipients receiving mycophenolic acid [9-11]. Inhibition of hIMPDH2 has become an important strategy in the treatment of diseases related to immunosuppression, cancer, and viral and parasitic infections [12-16]. Although it has long been the belief that chemotherapy would be improved with selective inhibition of hIMPDH2, this view has recently been challenged by the surprising observation that hIMPDH1 is also an antiangiogenic target [17].

The research of hIMPDH inhibitors is of great significance in providing potentially therapeutic effects against this target for disease intervention. There are three types of hIMPDH inhibitors: (i) IMP site inhibitors that occupy the binding position of the natural substrate IMP; (ii) $\mathrm{NAD}^{+}$site inhibitors that occupy the site of the $\mathrm{NAD}^{+} / \mathrm{NADH}$ cofactor; and (iii) allosteric site inhibitors that bind to a site remote from the IMP and $\mathrm{NAD}^{+}$binding pockets. Many researchers are interested in developing $\mathrm{NAD}^{+}$site inhibitors, and novel inhibitors of hIMPDH have been reported in the last decade [18]. For example, mycophenolate mofetil (MMF or Cellcept), which is a prodrug of mycophenolic acid (MPA), is an uncompetitive hIMPDH inhibitor that has been approved for the prevention of acute rejection in heart, kidney, or 
pancreas transplantations when used in combination with cyclosporine A $[19,20]$. However, an unfavorable gastrointestinal toxicity tolerability profile limits the drug's potential for the treatment of other autoimmune disorders. To overcome the limitations of MPA, Vertex developed a series of phenyl-oxazole urea hIMPDH inhibitors using structurebased drug design and high-throughput screening, among which VX-497 has been in phase II development for the hepatitis $\mathrm{C}$ virus (HCV) infection [21]. In addition, tiazofurin has been found to possess both antiviral and antiproliferative activities [22, 23]. Several compounds, such as quinolones $[24,25]$, amides [26], and indoles [27, 28], have been reported to possess potent hIMPDH inhibition activities. However, safety and selectivity are still deficient, and there is a continuing effort to develop novel hIMPDH inhibitors.

The pharmacophore model can be used to elucidate how diverse ligands bind to receptor sites and it can predict potential chemical interactions between ligands and a receptor. In addition, this model can be used to discover potent inhibitors of the target protein from selected database [29-31]. In this study, common feature pharmacophore modeling was used to uncover novel hIMPDH inhibitors from the ZINC database. Structure-based docking was then performed to analyze the binding modes and affinities of the identified compounds that show promise as hIMPDH inhibitors. Finally, interactions between IMPDH and the potential inhibitors were described in detail, with the aim to design novel drug candidates of hIMPDH.

\section{Methods}

The common feature pharmacophore model was generated using the Common Feature Pharmacophore Generation protocol in the Discovery Studio 3.0 software program (DS 3.0). Database screening was implemented using the Ligand Profiler Protocol in DS 3.0 [32]. Docking studies were performed with the glide module in the Schrödinger 2014 software program [33].

\subsection{Pharmacophore Generation and Validation}

2.1.1. Ligand Preparation. A set of ligands occupying the hIMPDH NAD ${ }^{+}$site with known inhibitory activities were collected from the literature to establish a common feature pharmacophore. All of the structures were constructed in DS 3.0. The most important step in pharmacophore modeling is the selection of suitable inhibitors that constitute the training set. For this purpose, 22 active hIMPDH inhibitors with diverse scaffolds [24, 27, 34-42] (Figure 1) were selected as the training set and generated using parameters from the CHARMm force field. All of the structures were minimized using the Steepest Descent algorithm, followed by the Conjugate Gradient and Adopted Basis Newton-Raphson algorithms, with convergence gradient values of $0.1 \mathrm{kcal} \cdot \mathrm{mol}^{-1}$, $0.01 \mathrm{kcal} \cdot \mathrm{mol}^{-1}$ and $0.001 \mathrm{kcal} \cdot \mathrm{mol}^{-1}$, respectively. A multiconformer database was generated using the poling algorithm with an energy threshold of $20 \mathrm{kcal} \cdot \mathrm{mol}^{-1}$ and a maximum of 255 conformers per molecule.
TABLE 1: Bioactivity of compounds in the training set from literature.

\begin{tabular}{|c|c|c|c|}
\hline Compound & $K_{i}[\mathrm{nM}]$ & $\mathrm{IC}_{50}[\mathrm{nM}]$ & Reference \\
\hline 1 & & 12 & [34] \\
\hline 2 & & 15 & {$[34]$} \\
\hline 3 & & 11 & {$[34]$} \\
\hline 4 & 6 & & {$[35]$} \\
\hline 5 & 7 & & {$[36]$} \\
\hline 6 & 6 & & [37] \\
\hline 7 & & 30 & {$[38]$} \\
\hline 8 & & 19 & {$[38]$} \\
\hline 9 & & 18 & {$[38]$} \\
\hline 10 & & 30 & {$[38]$} \\
\hline 11 & & 17 & [39] \\
\hline 12 & & 11 & [39] \\
\hline 13 & & 17 & [39] \\
\hline 14 & & 19 & {$[40]$} \\
\hline 15 & & 5 & {$[24]$} \\
\hline 16 & & $<5$ & {$[24]$} \\
\hline 17 & & $<10$ & {$[24]$} \\
\hline 18 & & 11 & {$[24]$} \\
\hline 19 & & 6 & {$[24]$} \\
\hline 20 & & 13 & {$[41]$} \\
\hline 21 & & 7 & {$[42]$} \\
\hline 22 & & 30 & [27] \\
\hline
\end{tabular}

2.1.2. Pharmacophore Generation. Pharmacophore model generation was performed using the multiconformer database described above, with activity values listed in Table 1 . For the most potent inhibitors (Compounds 1, 3, 4, 5, 6, 12, 15, 16, 17, 18, 19, 20, and 21), the Principal and MaxOmitFeat values were set to 2 and 0 , respectively. For the moderate inhibitors (Compounds 2, 7, 8, 9, 10, 11, 13, 14, and 22), the Principal and MaxOmitFeat values were both assigned values of 1 . The H-bond acceptor (A), H-bond donor (D), hydrophobic aliphatic $(\mathrm{H})$, and hydrophobic aromatic $(\mathrm{Z})$ features were selected based on the chemical features of the ligands in the training set using the Feature Mapping protocol in DS 3.0. The value of Maximum Pharmacophore was set to 10 . The default Minimum Interfeature Distance value of $2.97 \AA$ was changed to $2.0 \AA$ so that the chemical features located close to each other would be considered when generating the pharmacophore. The minimum features were set to 4 , and the maximum features were set to 7 . Default values were used for all other parameters. From this approach, ten pharmacophore hypotheses were successfully generated. These models were validated with both the training set and the decoy set.

2.1.3. Pharmacophore Validation. The training set compounds were aligned to the ten generated pharmacophore hypotheses using the Ligand Profiler Protocol in DS 3.0. The Maximum Omitted Features option was set to -1 , and the Scale Fit Values were set to false. Default values were used for all other parameters. To confirm the quality of these models, the ten generated pharmacophore hypotheses were assessed by rank scores and fit values. Subsequently, some poor 


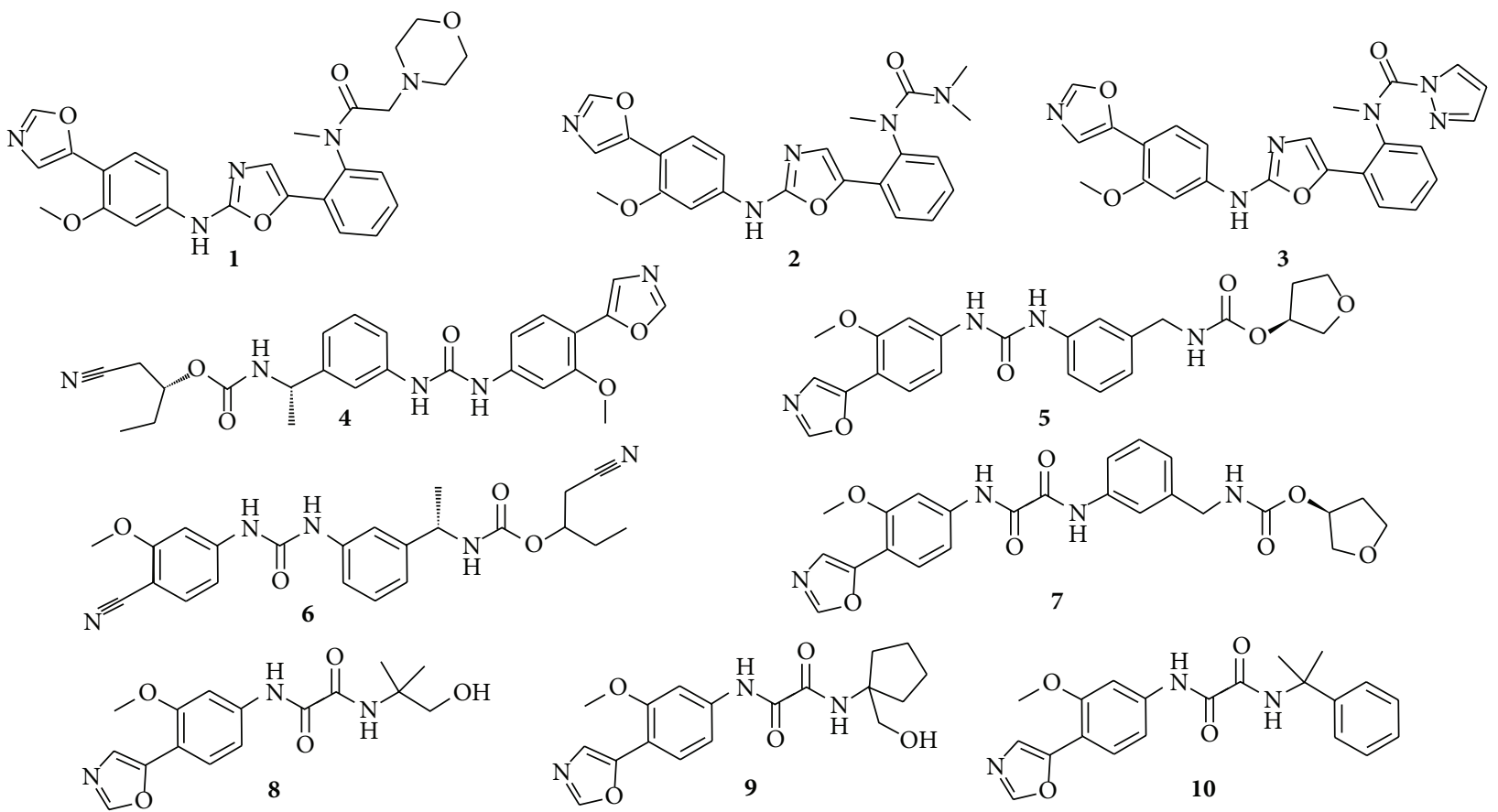<smiles>CCN1CCN(c2ccc(C(C)(C)NC(=O)c3cc4[nH]c5ccccc5c(=O)c4cc3F)cn2)CC1</smiles><smiles>COc1cc2[nH]c(-c3ccc4c(c3)C(=O)CCC4)cc(=O)c2cc1-c1cnco1</smiles>

12<smiles>CC(C)(NC(=O)c1cc2c(cc1F)NCCO2)c1cnc2[nH]c3ccccc3c(=O)c2c1</smiles>

13<smiles>COc1cc2[nH]c(=S)n(CCc3ccncc3)c(=O)c2cc1-c1cnco1</smiles>

20<smiles>COc1cc2[nH]c(-c3cccc(N4CCOCC4)c3)cc(=O)c2cc1-c1cnco1</smiles>

15<smiles>COc1cc2[nH]c(-c3ccc4c(c3)C(OC(=O)N(C)C)CC4)cc(=O)c2cc1-c1cnco1</smiles><smiles>CC(=O)OCC(=O)N(C)c1ccccc1-c1cnc(Nc2ccc3c(-c4cnco4)c[nH]c3c2)o1</smiles><smiles>COc1cc2[nH]c(-c3ccc(C)c(OCCN4CCOCC4)c3)cc(=O)c2cc1-c1cnco1</smiles>

16<smiles>COc1cc2[nH]c(-c3ccc4c(c3)C(N(C)C)CC4)cc(=O)c2cc1-c1cnco1</smiles><smiles>COc1cc2[nH]c(-c3csc4ccccc34)c(C=O)c2cc1-c1cnco1</smiles>

FIGURE 1: Structures of compounds 1-22 in the training set for the development of pharmacophore model.

models were rejected and the remaining pharmacophore models were assessed using another method. In general, pharmacophore models are used as 3D queries to search databases to discover novel and potent lead compounds. A validation of the generated pharmacophore model should be performed to determine whether the model is able to accurately differentiate between active and inactive compounds.
The Güner-Henry (GH) scoring method was used to validate the pharmacophore hypotheses $[43,44]$. This method quantifies the merit of the generated model by retrieving the active compounds from a database containing known active and inactive molecules. A decoy set containing 729 molecules was constructed to validate the generated pharmacophore. Of these 729 molecules, 29 molecules were known inhibitors of 
hIMPDH, whereas the other 700 molecules were obtained from the ZINC database using the Find Similar protocol in DS 3.0. The GH score method has been successfully applied to quantify the selectivity of the pharmacophore model and to discover activities from a decoy database. The GH score was calculated using the following formulae:

$$
\begin{gathered}
Y \%=\frac{H_{a}}{T_{a}} \times 100 \% \\
H_{t} \%=\frac{H_{a}}{H_{t}} \times 100 \% \\
E=\frac{H_{a} / H_{t}}{T_{a} / T} \\
\mathrm{GH}=\frac{H_{a}\left(3 H_{a}+H_{t}\right)}{4 H_{t} T_{a}}\left(1-\frac{H_{t}-H_{a}}{T-T_{a}}\right),
\end{gathered}
$$

where $H_{a}$ is the number of active hits, $T_{a}$ is the total number of actives in the decoy set, $H_{t}$ is the total number of hits including actives and decoy molecules, $T$ is the total number of molecules in the decoy set, $Y \%$ is the percentage of known active compounds obtained from the decoy set, $H_{t} \%$ is the percentage of known actives in the hits list, $E$ is the enrichment factor, and $\mathrm{GH}$ is the Güner-Henry score. The $\mathrm{GH}$ score ranging from 0.6 to 1 would indicate an optimal pharmacophore model.

2.2. Database Searching. Virtual screening of a chemical database often leads to the discovery of novel and potential lead compounds for further development. The optimal pharmacophore model (hypo-07) was used as the 3D query for screening the ZINC database, which contains 325,881 drug-like molecules. All screening experiments were performed using the Ligand Profiler Protocol in DS 3.0. During the screening process, we performed the virtual screening using the Best/Flexible conformational procedure, with a maximum of 255 conformations generated. The Maximum Omitted Features option was set to -1 , and the Scale Fit Values were set to false. Molecules that possessed all the desired pharmacophore features were considered to be hit compounds. The so-called "Lipinski's rule of five" was not applied to selected compounds because we wanted to prevent the removal of potential inhibitors. Those molecules that successfully passed these initial tests were selected for subsequent molecular docking analysis.

2.3. Molecular Docking. Docking study is a necessary step for picking out potential hits in virtual screening [45]. To investigate the detailed interactions between the virtual hits and IMPDH, the glide module in the Schrödinger software program was used to perform docking studies.

The 3D structure of IMPDH in complex with IMP and MPA (PDB ID: 1JR1) has been determined by X-ray diffraction. MPA inhibits IMPDH by acting as a replacement for the nicotinamide portion of the nicotinamide adenine dinucleotide cofactor $\left(\mathrm{NAD}^{+}\right)$and a catalytic water molecule. Our primary aim was to obtain $\mathrm{NAD}^{+}$site inhibitors; thus, the 1JR1 crystal structure was used for docking studies and the position of MPA was selected as the active site. The protein was prepared with the Protein Preparation Wizard (PrepWizard) in the Schrödinger software program. The location of MPA in the 1JR1 crystal structure was used to define the size and center of the receptor grid. An active site of $5 \AA$ was set around the MPA ligand. To prepare the protein, MPA was deleted, IMP was retained, hydrogen atoms were added, water molecules beyond $5 \AA$ from the groups were deleted, and the bond orders of the protein were adjusted and minimized up to $0.30 \AA$ root mean square deviation (RMSD). Then, the original conformation of the MPA was docked into the $\mathrm{NAD}^{+}$site of IMPDH using both rigid and flexible methods to validate the docking procedure. When the RMSD value ranges between 0 and 2, the program can be used for docking studies of other ligands. The ideal case is when the RMSD value is below 0.5. Finally, the extra precision (XP) mode and other default parameters of the glide module were used for the docking studies [46]. The compounds of the training set and the hit compounds were docked into the active site of IMPDH using the flexible docking strategy to predict potential inhibitors of IMPDH.

\section{Results}

3.1. Generation and Validation of the Common Feature Pharmacophore Model of hIMPDH Inhibitors. A pharmacophore, which is known as a powerful tool to identify novel compounds with good biological activity, can be established via a ligand-based method. The common feature pharmacophore generation protocol in DS 3.0 was employed with the training set compounds. As suggested by the Feature Mapping protocol, pharmacophore models were generated using the following features: H-bond acceptor (A), H-bond donor (D), hydrophobic_aliphatic group (H), and hydrophobic_aromatic group (Z). Ten pharmacophore hypotheses were obtained for further investigation based on the training set molecules. All ten pharmacophore hypotheses contain the above four chemical features. Statistical parameters of the generated pharmacophore models are listed in Table 2. Rank scores of the ten models range from $215.59 \mathrm{kcal} \cdot \mathrm{mol}^{-1}$ to $229.50 \mathrm{kcal} \cdot \mathrm{mol}^{-1}$. Direct and partial hit values of 1 and 0 indicate that the ligands are well mapped onto all of the chemical features of the model (i.e., there are no missing features). The max fit of all the hypotheses is 4 . Clearly, the fit values of hypotheses $03,04,07$, and 08 (i.e., hypo-03, $04,-07,-08)$ are higher than those of hypo-01, -02, -05, $06,-09$, and -10 . For this reason, the former four hypotheses were selected for further investigation (Table 3). However, on the basis of rank scores and fit values alone, we could not determine which pharmacophore hypothesis is the best model. Therefore, the Güner-Henry (GH) scoring method was used to identify the best pharmacophore model. A decoy database, which was used for pharmacophore identification and validation, was constructed to include 20 independent active hIMPDH inhibitors, 9 inactive hIMPDH inhibitors, and 700 inactive ligands from the ZINC database. Notably, all of the 20 active molecules were successfully identified by hypotheses 03,04 , and 07 , and the number of total hits 
TABLE 2: Top ten pharmacophore hypotheses generated by IMPDH inhibitors.

\begin{tabular}{lccccc}
\hline Hypo & Features & Rank & Direct hit $^{\mathrm{a}}$ & Partial hit $^{\mathrm{b}}$ & Max fit $^{\circ}$ \\
\hline 01 & ZHDA & 229.50 & 1111111111111101111111 & 0000000000000010000000 & 4 \\
02 & ZHDA & 229.42 & 1111111111111111111111 & 0000000000000000000000 & 4 \\
03 & ZHDA & 226.70 & 1111111111111111111111 & 0000000000000000000000 \\
04 & ZHDA & 225.26 & 1111110111111111111111 & 0000001000000000000000 \\
05 & ZHDA & 223.74 & 1111110011111111111111 & 0000001100000000000000 \\
06 & ZHDA & 219.08 & 1111110011111111111111 & 0000001100000000000000 & 4 \\
07 & ZHDA & 218.00 & 1111110011111111111111 & 0000001100000000000000 & 4 \\
08 & ZHDA & 218.10 & 1011111111011111111110 & 0100000000100000000001 & 4 \\
09 & ZHDA & 216.52 & 1011111011011110111111 & 0100000100100001000000 & 4 \\
10 & ZHDA & 215.59 & 1111110001110111111111 & 0000001110001000000000 & 4 \\
\hline
\end{tabular}

"Direct hit indicates whether " 1 " or not "0" a molecule in the training set mapped every pharmacophore feature in the hypothesis. ${ }^{b}$ Partial hit connotes whether " 1 " or not " 0 " a particular molecule in the training set mapped all but one pharmacophore feature in the hypothesis.

TABLE 3: The hit values of compounds in the training set mapping to hypotheses $03,04,07$, and 08 .

\begin{tabular}{lcccc}
\hline Comp. & Hypo-03 & Hypo-04 & Hypo-07 & Hypo-08 \\
\hline $\mathbf{1}$ & 3.208 & 3.265 & 3.288 & 3.156 \\
$\mathbf{2}$ & 3.165 & 3.303 & 3.061 & 3.880 \\
$\mathbf{3}$ & 3.665 & 3.390 & 3.502 & 3.775 \\
$\mathbf{4}$ & 3.980 & 3.980 & 4.000 & 3.887 \\
$\mathbf{5}$ & 3.999 & 3.999 & 3.988 & 3.784 \\
$\mathbf{6}$ & 3.749 & 3.660 & 3.605 & 3.583 \\
$\mathbf{7}$ & 3.210 & 3.408 & 3.362 & 3.528 \\
$\mathbf{8}$ & 2.992 & 2.980 & 2.997 & 3.029 \\
$\mathbf{9}$ & 2.990 & 2.994 & 2.977 & 3.648 \\
$\mathbf{1 0}$ & 3.578 & 3.369 & 3.661 & 3.078 \\
$\mathbf{1 1}$ & 2.669 & 2.776 & 2.780 & 2.865 \\
$\mathbf{1 2}$ & 2.658 & 2.7281 & 2.764 & 2.830 \\
$\mathbf{1 3}$ & 2.436 & 2.750 & 2.825 & 3.146 \\
$\mathbf{1 4}$ & 2.889 & 3.161 & 2.898 & 2.255 \\
$\mathbf{1 5}$ & 3.189 & 3.086 & 3.428 & 3.999 \\
$\mathbf{1 6}$ & 3.086 & 3.133 & 3.444 & 3.262 \\
$\mathbf{1 7}$ & 3.160 & 3.031 & 3.338 & 3.475 \\
$\mathbf{1 8}$ & 3.115 & 3.032 & 3.277 & 3.652 \\
$\mathbf{1 9}$ & 3.100 & 3.099 & 3.261 & 3.745 \\
$\mathbf{2 0}$ & 3.868 & 3.796 & 3.771 & 2.998 \\
$\mathbf{2 1}$ & 3.613 & 3.239 & 3.343 & 3.307 \\
$\mathbf{2 2}$ & 2.987 & 3.046 & 3.096 & 2.914 \\
\hline
\end{tabular}

was 66,75 , and 35 for each hypothesis, respectively. Other statistical parameters, such as yield of actives, hit rate of actives, enrichment factor, and GH score, are presented in Table 4 . The GH score is one of the standards used to assess the quality of generated models. In this regard, hypothesis 07 (hypo-07) demonstrates the highest GH score value of 0.67 , which indicates a strong capability to choose the active rather than inactive molecules from the database.

The 3D spatial relationship and geometric parameters of hypo-07 are shown in Figure 2(a). Figures 2(b)-2(d) describe the alignment of hypo-07 with three different inhibitors (i.e., compounds $5\left(K_{i}=7 \mathrm{nM}\right), 20\left(\mathrm{IC}_{50}=13 \mathrm{nM}\right)$, and $22\left(\mathrm{IC}_{50}=\right.$ $30 \mathrm{nM})$, resp.). Clearly, the four pharmacophore features map very well onto compounds $\mathbf{5}$ and $\mathbf{2 0}$, which have previously been shown to be potent inhibitors. In addition, other potent inhibitors such as compounds $1,3,4,6,15,16,17,18,19,20$, and 21, but not compound 12, present good hit values (Table $3)$. For the moderately active compound 22, the H-bond acceptor and hydrophobic_aliphatic pharmacophore features could match with the ligand but the H-bond donor and the hydrophobic_aromatic features did not. These results reveal that a selected pharmacophore model might be capable of predicting the activity of compounds. Consequently, hypo-07 was applied for further studies to identify novel inhibitors.

3.2. Database Screening. The best pharmacophore model (i.e., hypo-07) was used to search the ZINC database, which contains 325,881 compounds, for new hIMPDH inhibitor candidates. Among the 325,881 compounds, 1566 compounds passed the initial screening and were mapped onto the pharmacophore model, which included some compounds that are structurally similar to existing hIMPDH inhibitors and some novel scaffolds. Sixty-nine hits were selected based on the fit value. All of these compounds have common features that can align to the required pharmacophore sites of hypo-07. The 69 hit compounds were exported as an.sdf file and subjected to further analysis using molecular docking to avoid possible false positive hits from the virtual screening process.

\subsection{Molecular Docking Studies of hIMPDH Inhibitors and Hit Compounds}

3.3.1. Docking Validation. In this study, the validation of the docking procedure was performed by redocking cocrystallized MPA into the active site of IMPDH using both the rigid and flexible methods of the glide module. We found that the redocked MPA reproduced the binding pose with glide scores of $-6.65 \mathrm{kcal} \cdot \mathrm{mol}^{-1}$ and $-6.59 \mathrm{kcal} \cdot \mathrm{mol}^{-1}$, respectively. The RMSD of the cocrystallized and experimental poses was analyzed, and the values of the two methods were $0.54 \AA$ and 
TABLE 4: The evaluation of ligand-based pharmacophore model using the Güner-Henry scoring method.

\begin{tabular}{lcccc}
\hline & Hypo-03 & Hypo-04 & Hypo-07 & Hypo-08 \\
\hline Total number of molecules in the decoy set $(T)$ & 729 & 729 & 729 & 20 \\
Total number of actives in the decoy set $\left(T_{a}\right)$ & 20 & 20 & 20 & 20 \\
Active hits $\left(H_{a}\right)$ & 20 & 75 & 35 & 15 \\
Total hits $\left(H_{t}\right)$ & 66 & $100 \%$ & 54 & $75 \%$ \\
Yield of actives $(Y \%)$ & $100 \%$ & $26.67 \%$ & $57.14 \%$ & 20.83 \\
Hit rate of actives $\left(H_{t} \%\right)$ & $30.30 \%$ & 9.72 & 0.67 \\
Enrichment factor $(E)$ & 11.05 & 0.41 & 0.13 \\
Goodness of hit score $(\mathrm{GH})$ & 0.45 & & 0.50 \\
\hline
\end{tabular}

GH: Güner-Henry.

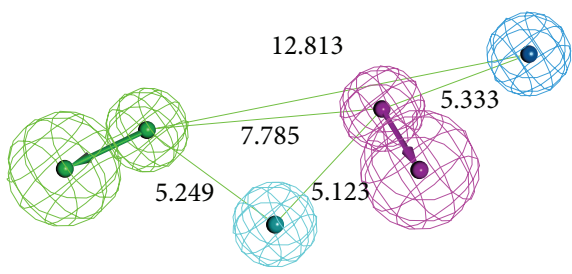

(a)

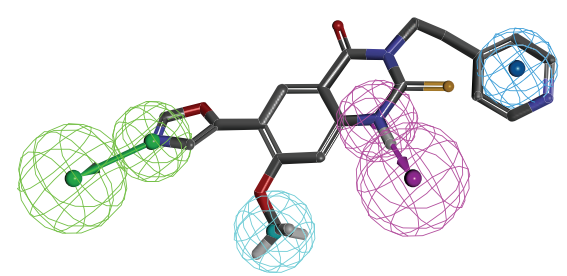

(c)

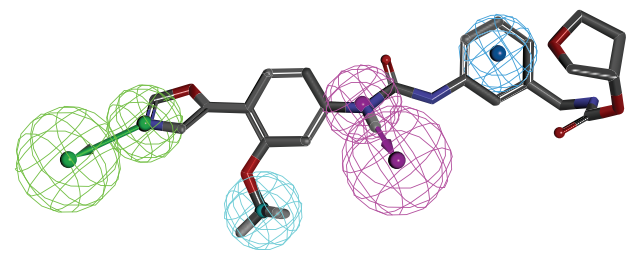

(b)

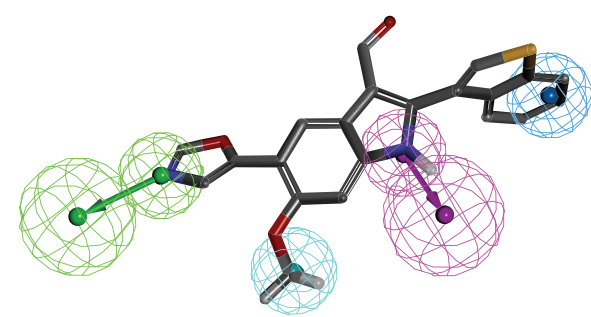

(d)

FIgURE 2: (a) 3D spatial relationship and geometric parameters of hypo-07. Pharmacophore features colored as follows: H-bond acceptor (green), H-bond donor (magenta), hydrophobic_aliphatic group (cyan) and hydrophobic_aromatic group (sky blue). (b) Hypo-07 mapping with active compound 5. (c) Hypo-07 mapping with active compound 20. (d) Hypo-07 mapping with moderate active compound 22.

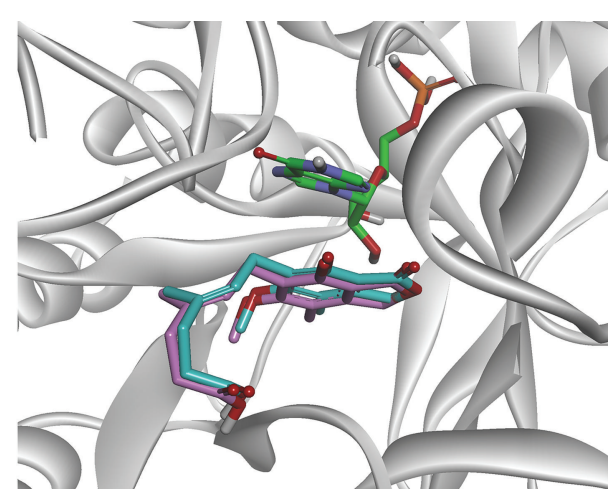

Figure 3: The alignment of redocked (pink) and cocrystallized ligand (cyan) in the active site of IMPDH. The green ligand is IMP.

$0.43 \AA$, respectively. These results showed that docking simulations reproduced the crystal complexes very well. The alignment of the cocrystallized ligand (green) and redocked ligand (pink) is shown in Figure 3. The glide XP program was suitable for further studying the binding pose of the novel hits.
3.3.2. The Docking Results. The nonbond interactions were examined using the similarly labeled tool in the Discovery Studio 4.0 Visualizer software program; various interactions between the inhibitors and IMPDH were examined, including traditional hydrogen bonding interactions, carbonhydrogen bonding interactions, pi-donor hydrogen bonding interactions, and hydrophobic interactions. According to the docking results, we can see that the interface points of IMPDH include interactions from Pro69, Met70, Asp71, His92, His93, Asn94, Cys95, Ala249, Gly251, Thr252, His253, Asp256, Arg259, Asp274, Ser275, Gln277, Asn303, Arg322, Gly324, Met325, Gly326, Cys327, Gly328, Cys331, Ile332, Thr333, Gln334, Asp364, Met414, Gly415, Ala419, Met420, and Gln441. On the basis of these results, some active sites and residues were identified from the IMPDH complex.

First, the docking results of MPA and compound 5 (i.e., VX-497) of the training set are described. On one hand, research on the known active ligands can help us to identify the suitable binding modes of the inhibitors in the $\mathrm{NAD}^{+}$site of IMPDH. On the other hand, the analysis of interactions between VX-497 and IMPDH can rationalize the pharmacophore model from another aspect. 


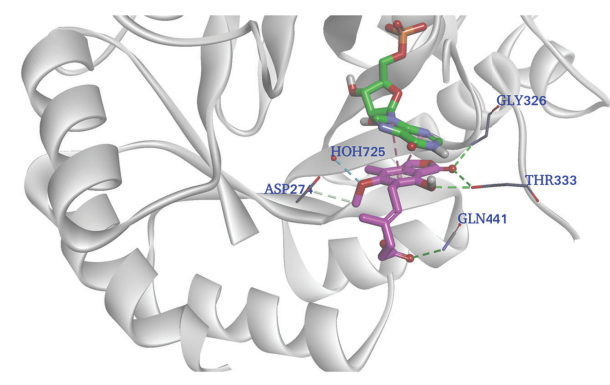

(a)

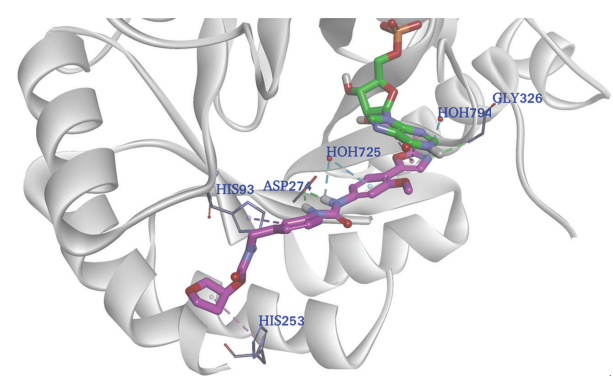

(b)

FIGURE 4: Binding patterns of potent inhibitors with IMPDH. The secondary structure of IMPDH is shown as a white rainbow. Residues in the active site are shown in gray lines and labelled as amino acid names. IMP is shown in green stick. The H-bonding interactions are shown using green dotted lines, weak H-bonding interaction is shown using pale-cyan dotted lines, and hydrophobic interactions are shown using pink dotted lines. (a) The binding mode of cocrystallized ligand (MPA, magenta) in the active site of IMPDH. (b) The binding mode of compound 5 (VX-497, magenta) in the active site of IMPDH.

MPA exhibits various interactions with the amino acid residues of the active site, as shown in Figure 4(a). The interactions between MPA and IMPDH active site residues were identical with the cases reported by Sintchak and Nimmesgern [47]. The results suggest that the carbonyl group of the lactone ring bonds to IMPDH with its oxygen atom to form hydrogen bonding interactions with the backbone $\mathrm{NH}$ moiety of Gly326 and the $\mathrm{OH}$ group of Thr333. At the same time, the oxygen atom of the hydroxyl group of MPA forms a hydrogen bonding interaction with the $\mathrm{OH}$ group of Thr333. The carbonyl group of $\mathrm{COOH}$ forms a hydrogen bonding interaction with the $\mathrm{NH}$ moiety of Gln441. The oxygen atom $(\mathrm{O} 3)$ of the methoxy group forms a weak waterhydrogen bonding interaction with $\mathrm{H}_{2} \mathrm{O} 725$ and the carbon atom (C8) of the methoxy group is involved in a carbonhydrogen bonding interaction with Asp274. IMP, which is another ligand of IMPDH, was retained during docking studies because its purine ring forms important $\pi-\pi$ hydrophobic interactions with MPA.

The binding models between VX-497 and IMPDH active site residues are similar to most of the interactions reported by Sintchak and Nimmesgern [47]. The docking analysis demonstrates that the oxazole of VX-497 bonds to IMPDH via its nitrogen atom to form a hydrogen bonding interaction with the NH moiety of Gly326. The same nitrogen atom also forms a water-hydrogen bonding interaction with $\mathrm{H}_{2} \mathrm{O} 794$. The benzene ring connected with oxazole makes a $\pi$-donor hydrogen bonding interaction with $\mathrm{H}_{2} \mathrm{O} 725$. The additional hydrogen bonding interactions are formed between the $\mathrm{NH}$ moiety of the urea group and Asp274. Notably, the nitrogen atom of oxazole and the $\mathrm{NH}$ moiety of the urea group serve as a $\mathrm{H}$-bond acceptor and a $\mathrm{H}$-bond donor, respectively, which is identical to the analogous features of the best pharmacophore model. In addition, another benzene ring of VX-497 forms a $\pi-\pi$ hydrophobic interaction with His93, corresponding to the aromatic hydrophobic feature of the pharmacophore model. At the same time, the tetrahydrofuran ring of the inhibitor forms a $\pi$-alkyl hydrophobic interaction with His 253 . The $\pi-\pi$ hydrophobic interactions between the inhibitor and the purine ring of IMP are also formed (Figure 4(b)).
TABLE 5: The glide score of hit compounds compared with MPA and VX-497.

\begin{tabular}{lcc}
\hline Comp. & Glide XP Gscore $\left(\mathrm{kcal} \cdot \mathrm{mol}^{-1}\right)$ & Glide energy \\
\hline (1) ZINC02090792 & -7.80 & -59.779 \\
(2) ZINC00048033 & -7.58 & -59.395 \\
(3) ZINC00822338 & -7.57 & -64.007 \\
(4) ZINC08714541 & -7.17 & -58.716 \\
(5) ZINC06662648 & -7.06 & -60.263 \\
(6) ZINC00686714 & -6.98 & -64.254 \\
(7) ZINC00648305 & -6.95 & -55.144 \\
(8) ZINC02081544 & -6.93 & -62.507 \\
(9) ZINC00668789 & -6.90 & -57.154 \\
(10) ZINC02776094 & -6.88 & -55.596 \\
(11) MPA & -6.59 & -54.289 \\
(12) VX497 & -7.56 & -60.157 \\
\hline
\end{tabular}

From the above analyses, we believe that the role of Gly326 is to serve as a H-bond donor, which is necessary for potent inhibitors. An interaction between Thr333 and the ligand is also important. In addition, the critical hydrogen bonding interaction between the urea $\mathrm{NH}$ moiety and Asp274 contributes to the high potency observed for VX-497. Some hydrophobic interactions also play important roles in improving the activities of compounds.

The sixty-nine hit compounds that were predicted to be positive using the pharmacophore screening procedure were subjected to molecular docking studies. The potential compounds were selected according to the glide scores and their interactions with amino acid residues. Notably, ten hit compounds (see Figure S1 in Supplementary Material available online at http://dx.doi.org/10.1155/2015/418767) were found to have good glide scores compared to MPA and VX-497 (Table 5). Here, the binding modes of the two identified compounds with the highest glide scores from the ZINC database are described. They are ZINC02090792 and ZINC00048033, which are used for subsequent characterization as potential inhibitors. 


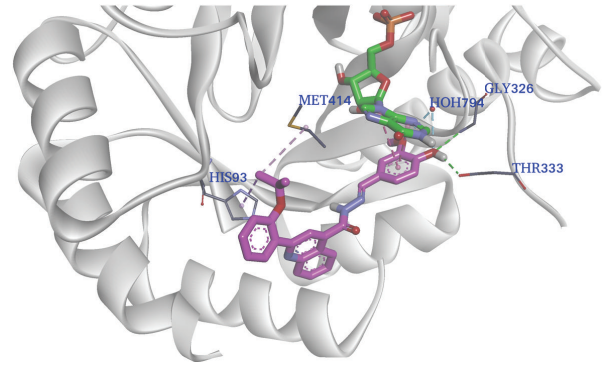

(a)

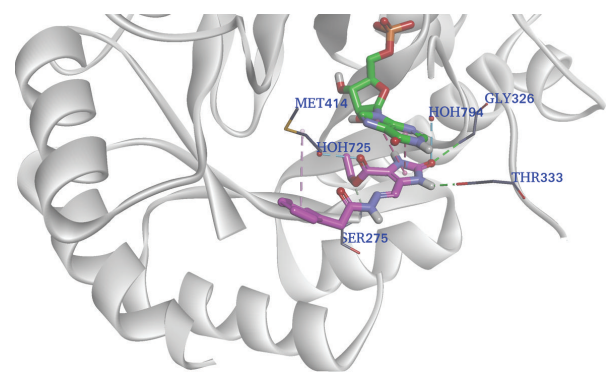

(c)

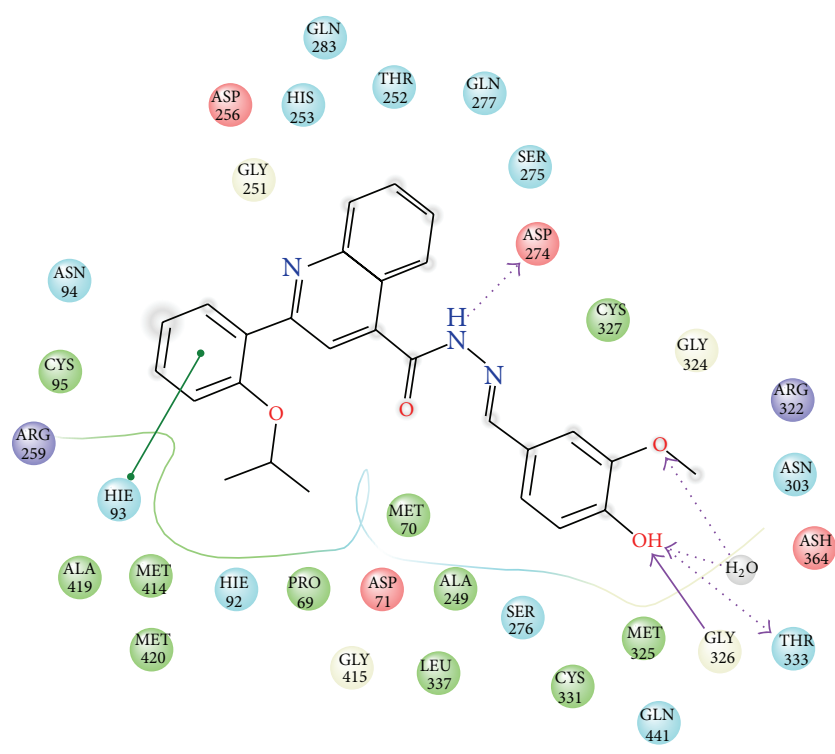

(b)

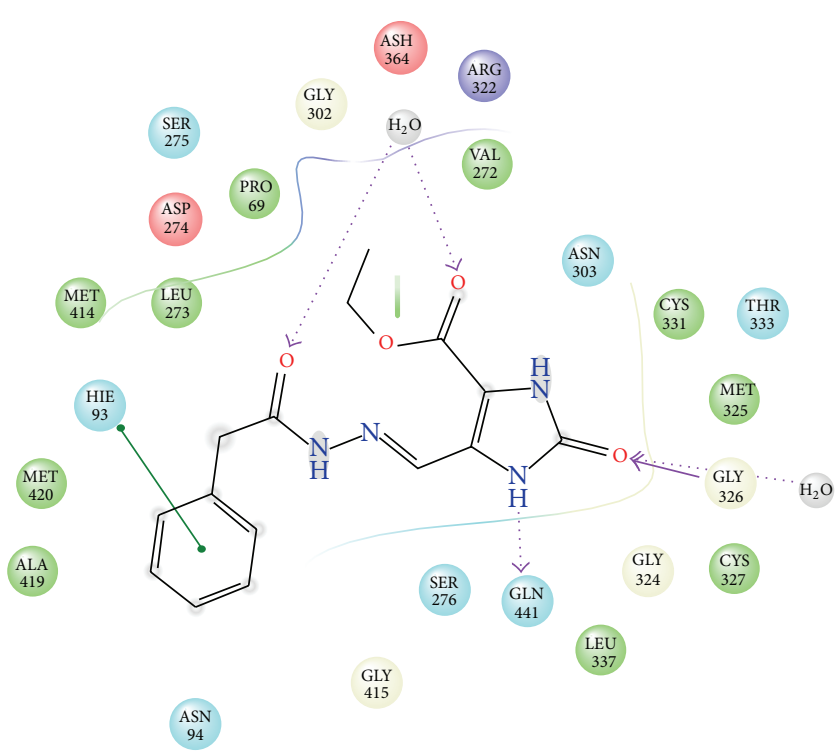

(d)

FIGURE 5: Binding mode and 2D interaction map of hit compounds. (a) The binding mode of ZINC02090792 in the active site of IMPDH. (b) The 2D interaction map of ZINC02090792 with the active site of IMPDH. (c) The binding mode of ZINC00048033 in the active site of IMPDH. (d) The 2D interaction map of ZINC00048033 with the active site of IMPDH.

The top virtual hit compound, ZINC02090792, shows similar binding interactions to MPA and VX-497 (Figure $5(\mathrm{a})$ ). The oxygen atom of the hydroxyl group serves as a $\mathrm{H}$-bond acceptor to form a hydrogen bonding interaction with the backbone NH moiety of Gly326, and the hydroxyl group serves as a H-bond donor to form a hydrogen bonding interaction with $T h r 333$. At the same time, both the hydroxyl and methoxy groups of ZINC02090792 form water-hydrogen bonding interactions with $\mathrm{H}_{2} \mathrm{O} 794$. From the 2D interaction map (Figure 5(b)) of ZINC02090792 in the active site of IMPDH, it is observed that $\mathrm{NH}$ serves as a $\mathrm{H}$-bond donor engaging in an interaction with Asp274. One carbon atom (C3) belonging to the methyl group of ZINC02090792 forms a $\pi$-alkyl hydrophobic interaction with His93, and it also makes an alkyl-alkyl hydrophobic interaction with Met414. In addition, the benzene ring connected with the hydroxyl and methoxy groups forms $\pi$ - $\pi$ hydrophobic interactions with the purine ring of IMP.

ZINC00048033 is another hit compound obtained using pharmacophore screening and docking validation. The interactions between ZINC00048033 and IMPDH are presented in Figures 5(c) and 5(d). The carbonyl group of the $1 \mathrm{H}$ imidazole-2-(3H)-one ring forms a hydrogen bonding interaction with the backbone $\mathrm{NH}$ moiety of Gly326, and it also makes a water-hydrogen bonding interaction with $\mathrm{H}_{2} \mathrm{O} 794$. Additionally, the $\mathrm{NH}$ group of $1 \mathrm{H}$-imidazole-2-(3H)-one ring 
serves as a $\mathrm{H}$-bond donor to form a hydrogen bonding interaction with Thr333. The carbonyl group that connects with the ethoxyl group forms a water-hydrogen interaction with $\mathrm{H}_{2} \mathrm{O} 725$. One carbon atom (C2) of ZINC00048033 engages in a carbon-hydrogen bonding interaction with Ser275. At the same time, a $\pi$-alkyl hydrophobic interaction between the benzene ring of ZINC00048033 and Met414 is observed. Similar to an active inhibitor of IMPDH, the $1 \mathrm{H}$-imidazole2 - $(3 H)$-one ring of ZINC00048033 forms $\pi-\pi$ hydrophobic interactions with the purine ring of IMP.

According to the above results, ZINC02090792 and ZINC00048033 form interactions with some key residues of IMPDH (i.e., Gly326, Thr333, and Asp274) in ways that are similar to the cocrystallized ligand MPA and the most potent ligand VX-497. Superimpositions of MPA on ZINC02090792 and ZINC00048033 bound to the active site are shown in Figure S2. Despite some changes in the interactions between active site residues and inhibitors (MPA, VX-497, ZINC02090792, and ZINC00048033), the conformations of these active site residues are virtually unchanged between the crystal structures of MPA or other three inhibitors bound to the active site of IMPDH. Consequently, ZINC02090792 and ZINC00048033 serve as lead compounds for developing novel hIMPDH inhibitors.

\section{Conclusions}

We have established a ligand-based pharmacophore model followed by virtual screening and molecular docking studies to discover novel hIMPDH inhibitors. The common feature pharmacophore models were generated using a training set that includes 22 active hIMPDH inhibitors. Ten hypotheses were obtained from this analysis, which all consisted of four features, including one hydrogen-bond acceptor, one hydrogen-bond donor, one hydrophobic_aliphatic group, and one hydrophobic_aromatic group. To confirm the quality of the pharmacophore models, a decoy test set was constructed and the Güner-Henry (GH) scoring method was used. The hypo-07 model was found to possess the highest GH score value (0.67). This result reveals that hypo07 is an optimal model to discriminate between active and inactive molecules within the database. The hypo-07 model was further used to screen the ZINC database to identify hIMPDH inhibitors, and sixty-nine potential candidates were selected. All the compounds can align to the required pharmacophore features of hypo-07. In addition, molecular docking was performed using the glide module to investigate the interactions between the potential candidates and IMPDH. From the docking studies, ten compounds were found to have higher glide scores than known active inhibitors. The top two hit compounds for IMPDH were ZINC02090792 and ZINC00048033, with corresponding glide scores of $-7.80 \mathrm{kcal} \cdot \mathrm{mol}^{-1}$ and $-7.58 \mathrm{kcal} \cdot \mathrm{mol}^{-1}$, respectively. Furthermore, the detailed interactions between the hit compounds and IMPDH were analyzed. ZINC02090792 forms hydrogen bonding interactions with Gly326, Thr333, Asp274, and $\mathrm{H}_{2} \mathrm{O} 794$. It also preserves a $\pi-\pi$ hydrophobic interaction with His93 and an alkyl-alkyl hydrophobic interaction with Met414. ZINC00048033 engages in hydrogen bonding interactions with Gly326, Thr333, $\mathrm{H}_{2} \mathrm{O} 725$, and $\mathrm{H}_{2}$ O794. At the same time, it forms a carbon-hydrogen bonding interaction with Ser275 and a $\pi$-alkyl hydrophobic interaction with Met414. The two potential hit compounds both form $\pi-\pi$ hydrophobic interactions with the purine ring of IMP. In conclusion, the identified hits serve as lead compounds for developing potential hIMPDH inhibitors. Derivatives of these two hits have been synthesized and an evaluation of their biological activity is now in progress.

\section{Conflict of Interests}

The authors declare that there is no conflict of interests regarding the publication of this paper.

\section{Authors' Contribution}

Rui-Juan Li and Ya-Li Wang contributed equally to this work.

\section{Acknowledgment}

The research was financially supported by the National Natural Science Foundation of China (Grant 21372157).

\section{References}

[1] M. D. Sintchak, M. A. Fleming, O. Futer et al., "Structure and mechanism of inosine monophosphate dehydrogenase in complex with the immunosuppressant mycophenolic acid," Cell, vol. 85, no. 6, pp. 921-930, 1996.

[2] L. Hedstrom, "IMP dehydrogenase: structure, mechanism, and inhibition," Chemical Reviews, vol. 109, no. 7, pp. 2903-2928, 2009.

[3] E. Huberman, D. Glesne, and F. Collart, "Regulation and role of inosine $-5^{\prime}$-monophosphate dehydrogenase in cell replication, malignant transformation, and differentiation," Advances in Experimental Medicine and Biology, vol. 370, pp. 741-746, 1994.

[4] M. Nagai, Y. Natsumeda, Y. Konno, R. Hoffman, S. Irino, and G. Weber, "Selective up-regulation of type II inosine $5^{\prime}$ monophosphate dehydrogenase messenger RNA expression in human leukemias," Cancer Research, vol. 51, no. 15, pp. 38863890, 1991.

[5] A. Zimmermann, J. J. Gu, J. Spychala, and B. S. Mitchell, "Inosine monophosphate dehydrogenase expression: transcriptional regulation of the type I and type II genes," Advances in Enzyme Regulation, vol. 36, pp. 75-84, 1996.

[6] A. G. Zimmermann, K. L. Wright, J. P.-Y. Ting, and B. S. Mitchell, "Regulation of inosine- 5 '-monophosphate dehydrogenase type II gene expression in human $\mathrm{T}$ cells. Role for a novel $5^{\prime}$ palindromic octamer sequence," Journal of Biological Chemistry, vol. 272, no. 36, pp. 22913-22923, 1997.

[7] A. G. Zimmermann, J. J. Gu, J. Laliberté, and B. S. Mitchell, "Inosine- 5 '-monophosphate dehydrogenase: regulation of expression and role in cellular proliferation and Tymphocyte activation," Progress in nucleic acid research and molecular biology, vol. 61, pp. 181-209, 1998.

[8] J. Jain, S. J. Almquist, P. J. Ford et al., "Regulation of inosine monophosphate dehydrogenase type I and type II isoforms in human lymphocytes," Biochemical Pharmacology, vol. 67, no. 4, pp. 767-776, 2004. 
[9] J. Wang, A. Zeevi, S. Webber et al., "A novel variant L263F in human inosine $5^{\prime}$-monophosphate dehydrogenase 2 is associated with diminished enzyme activity," Pharmacogenetics and Genomics, vol. 17, no. 4, pp. 283-290, 2007.

[10] M.-E. F. Mohamed, R. F. Frye, and T. Y. Langaee, "Interpopulation variation frequency of human inosine $5^{\prime}$-monophosphate dehydrogenase type II (IMPDH2) genetic polymorphisms," Genetic Testing, vol. 12, no. 4, pp. 513-516, 2008.

[11] T.-Y. Wu, Y. Peng, L. L. Pelleymounter et al., "Pharmacogenetics of the mycophenolic acid targets inosine monophosphate dehydrogenases IMPDH1 and IMPDH2: gene sequence variation and functional genomics," British Journal of Pharmacology, vol. 161, no. 7, pp. 1584-1598, 2010.

[12] K. Felczak and K. W. Pankiewicz, "Rehab of NAD(P)-dependent enzymes with NAD(P)-based inhibitors," Current Medicinal Chemistry, vol. 18, no. 13, pp. 1891-1908, 2011.

[13] L. Chen, R. Petrelli, K. Felczak et al., "Nicotinamide adenine dinucleotide based therapeutics," Current Medicinal Chemistry, vol. 15, no. 7, pp. 650-670, 2008.

[14] S. B. Braun-Sand and M. Peetz, "Inosine monophosphate dehydrogenase as a target for antiviral, anticancer, antimicrobial and immunosuppressive therapeutics," Future Medicinal Chemistry, vol. 2, no. 1, pp. 81-92, 2010.

[15] S. K. Gorla, N. N. McNair, G. Yang et al., "Validation of IMP dehydrogenase inhibitors in a mouse model of cryptosporidiosis," Antimicrobial Agents and Chemotherapy, vol. 58, no. 3, pp. 1603-1614, 2014.

[16] L. Hedstrom, G. Liechti, J. B. Goldberg, and D. R. Gollapalli, "The antibiotic potential of prokaryotic IMP dehydrogenase inhibitors," Current Medicinal Chemistry, vol. 18, no. 13, pp. 1909-1918, 2011.

[17] C. R. Chong, D. Z. Qian, F. Pan et al., "Identification of type 1 inosine monophosphate dehydrogenase as an antiangiogenic drug target," Journal of Medicinal Chemistry, vol. 49, no. 9, pp. 2677-2680, 2006.

[18] R. Petrelli, P. Vita, I. Torquati et al., "Novel inhibitors of inosine monophosphate dehydrogenase in patent literature of the last decade," Recent Patents on Anti-Cancer Drug Discovery, vol. 8, no. 2, pp. 103-125, 2013.

[19] M. D. Vu, S. Qi, D. Xu et al., "Synergistic effects of mycophenolate mofetil and sirolimus in prevention of acute heart, pancreas, and kidney allograft rejection and in reversal of ongoing heart allograft rejection in the rat," Transplantation, vol. 66, no. 12, pp. 1575-1580, 1998.

[20] A. H. Miladipour, A. J. Ghods, and H. Nejadgashti, "Effect of mycophenolate mofetil on the prevention of acute renal allograft rejection," Transplantation Proceedings, vol. 34, no. 6, pp. 2089-2090, 2002.

[21] "Phase II clinical trial of VX-497 for HCV infection begins," AIDS Patient Care STDS, vol. 12, no. 12, p. 944, 1998.

[22] A. Panattoni, F. D’Anna, and E. Triolo, "Antiviral activity of tiazofurin and mycophenolic acid against Grapevine Leafrollassociated Virus 3 in Vitis vinifera explants," Antiviral Research, vol. 73, no. 3, pp. 206-211, 2007.

[23] K. Kiguchi, F. R. Collart, C. Henning-Chubb, and E. Huberman, "Cell differentiation and altered IMP dehydrogenase expression induced in human T-lymphoblastoid leukemia cells by mycophenolic acid and tiazofurin," Experimental Cell Research, vol. 187, no. 1, pp. 47-53, 1990.

[24] T. G. M. Dhar, S. H. Watterson, P. Chen et al., "Quinolone-based IMPDH inhibitors: introduction of basic residues on ring D and
SAR of the corresponding mono, di and benzofused analogues," Bioorganic and Medicinal Chemistry Letters, vol. 13, no. 3, pp. 547-551, 2003.

[25] S. H. Watterson, M. Carlsen, T. G. M. Dhar et al., "Novel inhibitors of IMPDH: a highly potent and selective quinolone-based series," Bioorganic and Medicinal Chemistry Letters, vol. 13, no. 3, pp. 543-546, 2003.

[26] S. H. Watterson, C. Liu, T. G. M. Dhar et al., "Novel amidebased inhibitors of inosine $5^{\prime}$-monophosphate dehydrogenase," Bioorganic and Medicinal Chemistry Letters, vol. 12, no. 20, pp. 2879-2882, 2002.

[27] S. H. Watterson, T. G. M. Dhar, S. K. Ballentine et al., "Novel indole-based inhibitors of IMPDH: introduction of hydrogen bond acceptors at indole C-3," Bioorganic and Medicinal Chemistry Letters, vol. 13, no. 7, pp. 1273-1276, 2003.

[28] R. E. Beevers, G. M. Buckley, N. Davies et al., "Novel indole inhibitors of IMPDH from fragments: synthesis and initial structure-activity relationships," Bioorganic and Medicinal Chemistry Letters, vol. 16, no. 9, pp. 2539-2542, 2006.

[29] W. Tai, T. Lu, H. Yuan et al., "Pharmacophore modeling and virtual screening studies to identify new c-Met inhibitors," Journal of Molecular Modeling, vol. 18, no. 7, pp. 3087-3100, 2012.

[30] Y.-L. Wang, C.-Y. Lin, K.-C. Shih, J.-W. Huang, and C.-Y. Tang, "Design checkpoint kinase 2 inhibitors by pharmacophore modeling and virtual screening techniques," Bioorganic and Medicinal Chemistry Letters, vol. 23, no. 23, pp. 6286-6291, 2013.

[31] C.-Y. Lin and Y.-L. Wang, "Novel design strategy for checkpoint kinase 2 inhibitors using pharmacophore modeling, combinatorial fusion, and virtual screening," BioMed Research International, vol. 2014, Article ID 359494, 13 pages, 2014.

[32] Accelrys Inc, "Discovery Studio User manual," Discovery Studio Version 3.0, 2011.

[33] Schrödinger LLC, "Schrödinger user manuals," Glide Version 9.3, 2014.

[34] T. G. M. Dhar, Z. Shen, J. Guo et al., "Discovery of N-[2-[2-[[3methoxy-4-(5-oxazolyl)phenyl]amino]-5-oxazolyl]phenyl]-Nmethyl-4-morpholineacetamide as a novel and potent inhibitor of inosine monophosphate dehydrogenase with excellent in vivo activity," Journal of Medicinal Chemistry, vol. 45, no. 11, pp. 2127-2130, 2002.

[35] D. Floryk and T. C. Thompson, "Antiproliferative effects of AVN944, a novel inosine 5-monophosphate dehydrogenase inhibitor, in prostate cancer cells," International Journal of Cancer, vol. 123, no. 10, pp. 2294-2302, 2008.

[36] J. Jain, S. J. Almquist, D. Shlyakhter, and M. W. Harding, "VX497: a novel, selective IMPDH inhibitor and immunosuppressive agent," Journal of Pharmaceutical Sciences, vol. 90, no. 5, pp. 625-637, 2001.

[37] J. Jain, S. J. Almquist, A. D. Heiser et al., "Characterization of pharmacological efficacy of VX-148, a new, potent immunosuppressive inosine $5^{\prime}$-monophosphate dehydrogenase inhibitor," Journal of Pharmacology and Experimental Therapeutics, vol. 302, no. 3, pp. 1272-1277, 2002.

[38] H. H. Gu, E. J. Iwanowicz, J. Guo et al., "Novel diamide-based inhibitors of IMPDH," Bioorganic and Medicinal Chemistry Letters, vol. 12, no. 9, pp. 1323-1326, 2002.

[39] S. H. Watterson, P. Chen, Y. Zhao et al., "Acridone-based inhibitors of inosine $5^{\prime}$-monophosphate dehydrogenase: discovery and SAR leading to the identification of N-(2-(6-(4-ethylpiperazin-1-yl)pyridin-3-yl)propan-2-yl)-2-fluoro-9-oxo-9,10dihydroacridine-3-carboxamide (BMS-566419)," Journal of Medicinal Chemistry, vol. 50, no. 15, pp. 3730-3742, 2007. 
[40] T. Nakanishi, Y. Kozuki, Y. Eikyu et al., "In vitro and in vivo characterization of AS2643361, a novel and highly potent inosine 5/-monophosphate dehydrogenase inhibitor," European Journal of Pharmacology, vol. 674, no. 1, pp. 58-63, 2012.

[41] G. M. Buckley, N. Davies, H. J. Dyke et al., "Quinazolinethiones and quinazolinediones, novel inhibitors of inosine monophosphate dehydrogenase: synthesis and initial structure-activity relationships," Bioorganic and Medicinal Chemistry Letters, vol. 15, no. 3, pp. 751-754, 2005.

[42] T. G. M. Dhar, Z. Shen, C. A. Fleener et al., "The TosMIC approach to 3-(oxazol-5-yl) indoles: application to the synthesis of indole-based IMPDH inhibitors," Bioorganic and Medicinal Chemistry Letters, vol. 12, no. 22, pp. 3305-3308, 2002.

[43] R. P. Gangwal, N. R. Das, K. Thanki et al., "Identification of p38 $\alpha$ MAP kinase inhibitors by pharmacophore based virtual screening," Journal of Molecular Graphics and Modelling, vol. 49, pp. 18-24, 2014.

[44] S. Kalva, E. R. Azhagiya Singam, V. Rajapandian, L. M. Saleena, and V. Subramanian, "Discovery of potent inhibitor for matrix metalloproteinase- 9 by pharmacophore based modeling and dynamics simulation studies," Journal of Molecular Graphics and Modelling, vol. 49, pp. 25-37, 2014.

[45] G. Madhavi Sastry, M. Adzhigirey, T. Day, R. Annabhimoju, and W. Sherman, "Protein and ligand preparation: parameters, protocols, and influence on virtual screening enrichments," Journal of Computer-Aided Molecular Design, vol. 27, no. 3, pp. 221-234, 2013.

[46] R. A. Friesner, R. B. Murphy, M. P. Repasky et al., "Extra precision glide: docking and scoring incorporating a model of hydrophobic enclosure for protein-ligand complexes," Journal of Medicinal Chemistry, vol. 49, no. 21, pp. 6177-6196, 2006.

[47] M. D. Sintchak and E. Nimmesgern, "The structure of inosine $5^{\prime}$-monophosphate dehydrogenase and the design of novel inhibitors," Immunopharmacology, vol. 47, no. 2-3, pp. 163-184, 2000 . 


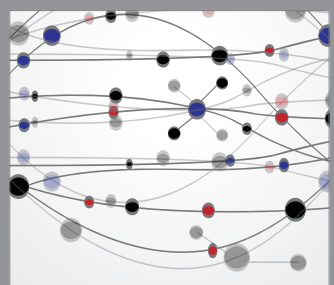

The Scientific World Journal
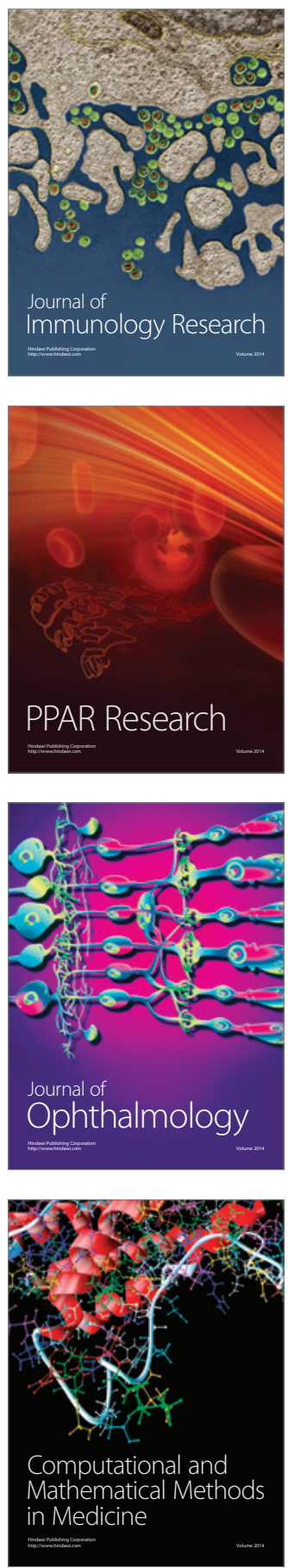

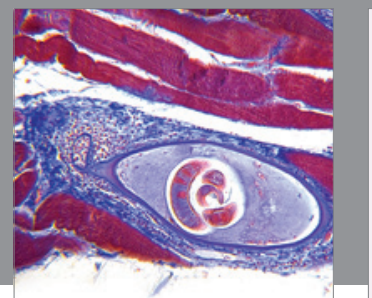

Gastroenterology

Research and Practice
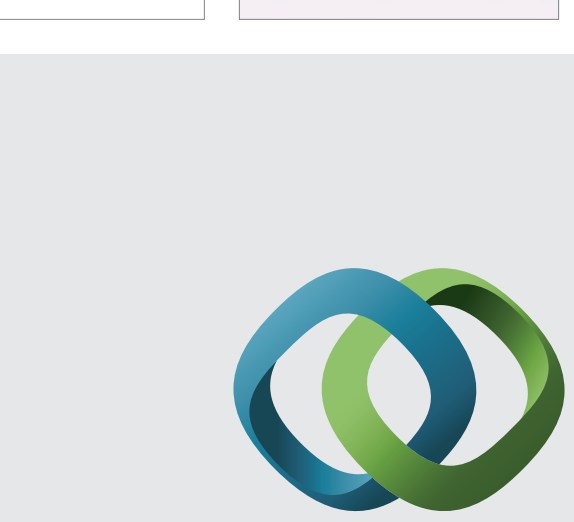

\section{Hindawi}

Submit your manuscripts at

http://www.hindawi.com
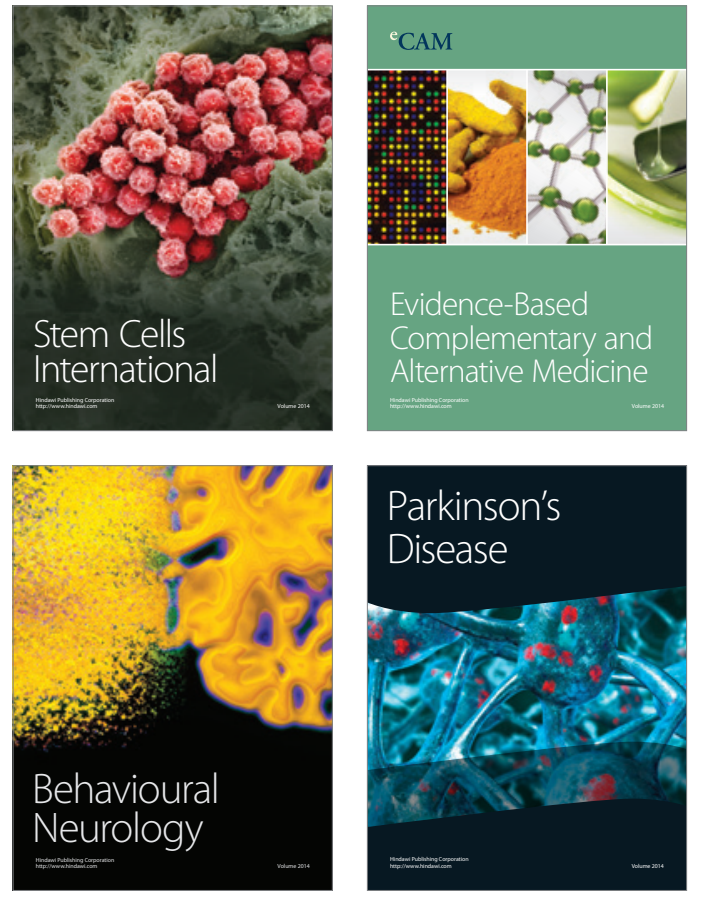
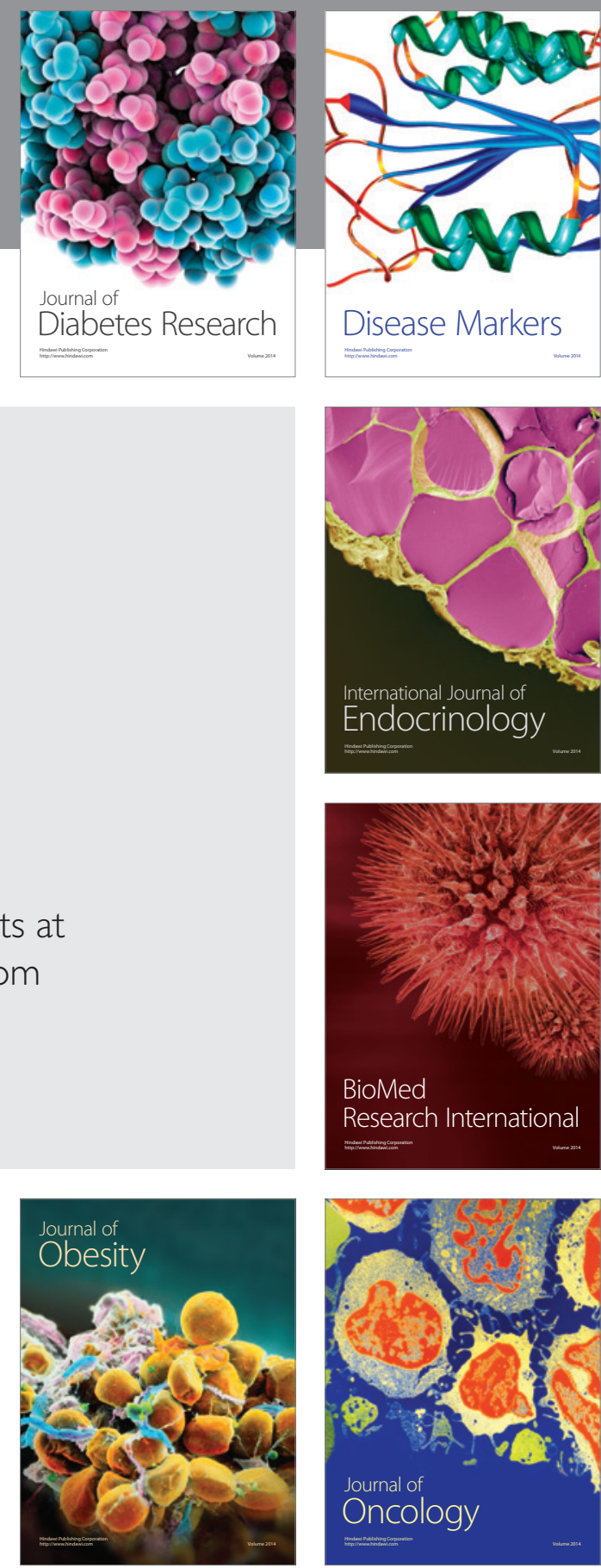

Disease Markers
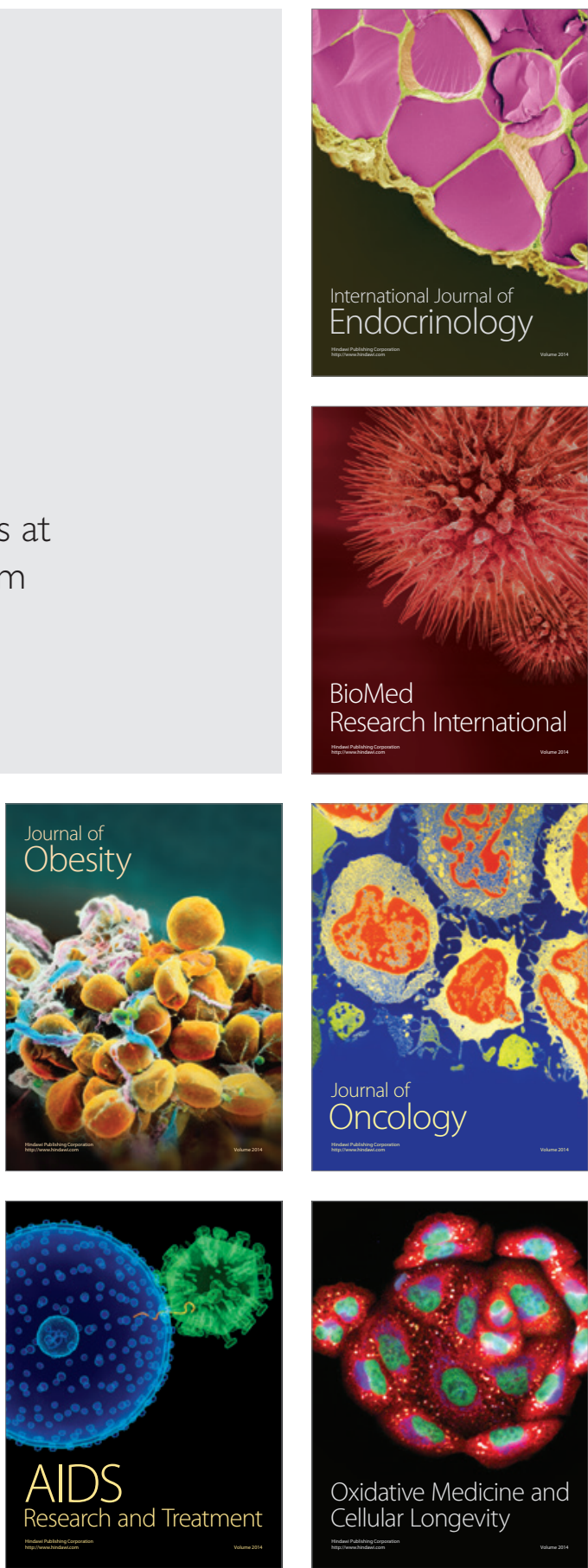